\title{
Estimativa da precipitação no Espírito Santo por intermédio de regressão polinomial
}

\author{
Marcel Carvalho ABREU ${ }^{1}$, Roberto Avelino CECÍLIO²*, Sidney Sara ZANETTI ${ }^{2}$, \\ Cecília Neves CATRINCK ${ }^{2}$
}

\author{
${ }^{1}$ Departamento de Ciências Ambientais, Universidade Federal Rural do Rio de janeiro, Seropédica, RJ, Brasil. \\ ${ }^{2}$ Departamento de Ciências Florestais e da Madeira, Universidade Federal do Espirito Santo, Jeronimo Monteiro, ES, Brasil \\ *E-mail: racecilio@gmail.com
}

Recebido em fevereiro/2018; Aceito em outubro/2018.

\begin{abstract}
RESUMO: A precipitação é um dos principais elementos da hidrologia, sendo uma variável de grande importância para a compreensão da dinâmica do ciclo hidrológico. Apesar da sua importância, a disponibilidade de dados hidroclimáticos é baixa. Dentre as alternativas para suprir a necessidade de informações da precipitação, a modelagem matemática é uma importante ferramenta que visa e sua estimativa. Assim, este trabalho avaliou as precipitações mensais e anuais de 110 estações pluviométricas do estado do Espírito Santo e avaliou o ajuste de modelos polinomiais de ordem 1 a 4 utilizando a longitude, latitude e altitude como variáveis explicativas para a previsão dessas precipitações. A precipitação no Espírito Santo mostrou variabilidade considerável, indicando grande influência do relevo, sendo observado também que localidades com maiores altitudes apresentaram maiores totais precipitados. A regressão polinomial de quarto grau se mostrou a mais adequada em representar as precipitações médias mensais e anual. Os ajustes foram considerados suficientes para representar as precipitações do Espírito Santo, com coeficientes de determinação superiores a 0,7 e erros percentuais absolutos médios entre 5,9\% e 16,6\%. Foi observada uma leve tendência dos modelos em subestimar os valores observados. De maneira geral, os meses do período seco, especialmente de maio a julho, obtiveram melhor desempenho dos modelos.
\end{abstract}

Palavras-chave: modelagem matemática, climatologia, hidrologia, chuva.

\section{Estimation of precipitation in the Espírito Santo State by polynomial regression}

\begin{abstract}
Precipitation is one of the main elements of hydrology, being a variable of great importance for understanding the dynamics of the hydrological cycle. Despite their importance, the availability of hydroclimatic data is low. Among the alternatives to meet the need for precipitation information, mathematical modeling is an important tool that aims its estimate. This work evaluated the monthly and annual rainfall of 110 rainfall gauges in the state of Espírito Santo and evaluated the adjustment of polynomial models of order 1 to 4 using longitude, latitude and altitude as explanatory variables to predict these precipitations. Precipitation in Espírito Santo showed considerable variability, indicating great influence of the relief, being observed that location with higher altitudes presented higher precipitated totals. The fourth-degree polynomial regression proved to be the most adequate to represent the mean monthly and annual precipitations. The adjustments were considered sufficient to represent the Espírito Santo precipitation, with coefficients of determination higher than 0.7 and mean absolute percentage errors between $5.9 \%$ and $16.6 \%$. A slight trend of the models was observed in underestimating the observed values. In general, the months of the dry period, especially from May to July, obtained better performance of the models.
\end{abstract}

Keywords: mathematical modeling, climatology, hydrology, rainfall.

\section{INTRODUÇÃO}

A precipitação é um dos principais elementos da ciência hidrológica, representando, dentro do ciclo hidrológico, uma variável imprescindível para a compreensão da dinâmica do meio físico. Além de apresentar suma importância na caracterização do clima de uma região, seu estudo permite previsões com melhores aproximações e tomadas de decisões mais confiáveis (ARAI et al., 2010).

Seu comportamento e entendimento são de grande relevância para estudos estratégicos associados ao planejamento e manejo de recursos hídricos (ASARIAN; WALKER, 2016; LI et al., 2017) e da agricultura (RANJITKAR et al., 2016; ZARO et al., 2014), especialmente em condições tropicais (MELLO; SILVA, 2009).
Devido à baixa disponibilidade de dados climáticos e hidrológicos, comparada a grandes extensões territoriais, em especial em países em desenvolvimento, muitos estudos têm sido realizados visando o mapeamento da precipitação (MELLO et al., 2007), com a utilização de diferentes técnicas de interpolação espacial (LOPES et al., 2016; LYRA et al., 2017).

Sabe-se que a precipitação possui alta variabilidade espaço-temporal, motivo pelo qual é considerada probabilística e aleatória pela hidrologia estocástica, assim, a modelagem matemática de seu comportamento é passível de ser feita com o ajuste de modelos lineares múltiplos (MARQUÍNEZ et al., 2003).

Com base no fato de que a altitude, a latitude e a longitude influenciam nas condições climáticas de uma região, é 
possível, aplicando-se técnicas estatísticas, ajustar equações de regressão polinomial múltipla para estimar os totais médios precipitados em função destas variáveis (GOMES et al., 2015; MELLO; SILVA, 2009).

As superfícies de tendência são interpoladores determinísticos globais que buscam modelar a variação em larga escala através de uma regressão múltipla, aproximando a superfície por um ajuste polinomial aos dados, entre os valores de atributo, as localizações geográficas e a altitude. A saída é uma função polinomial na qual o valor do atributo é expresso em função das coordenadas da superfície e sua altitude.

Neste contexto, a regressão polinomial (superfície de tendência) pode ser aplicada para se estimar as precipitações em locais específicos dentro da região de estudo.

Desta maneira, procurando suprir a necessidade de informação sobre os índices de precipitação no estado do Espírito Santo, a fim de possibilitar estudos mais precisos a fim de fundamentar a tomada de decisão em projetos, o presente trabalho objetivou ajustar modelos de regressão polinomial aplicados às precipitações médias mensais e anual para o estado do Espírito Santo.

\section{MATERIAL E MÉTODOS}

\section{1. Área em estudo e base de dados}

O estado do Espírito Santo possui uma área de aproximadamente $46.097 \mathrm{~km}^{2}$ e está situado na região sudeste, entre as latitudes $17^{\circ} 52^{\prime}$ e $21^{\circ} 19^{\prime} \mathrm{S}$ e as longitudes $39^{\circ} 38^{\prime}$ e $41^{\circ} 50^{\prime} \mathrm{O}$, totalmente incluído na região tropical e no bioma Mata Atlântica.

A base de dados utilizada foi obtida junto à Agência Nacional de Águas - ANA, por meio do sistema Hidroweb, e Instituto Capixaba de Pesquisa, Assistência Técnica e Extensão Rural - INCAPER. Foram trabalhados dados médios relativos aos totais precipitados nas escalas mensal e anual para o período de 1971 a 2011 (41 anos), originários de 110 estações pluviométricas localizadas no Espírito Santo e nas regiões circunvizinhas (Figura 1). A utilização dos postos pluviométricos dos estados vizinhos se deu com o objetivo de minimizar o efeito de borda no processo estimativa (CASTRO et al., 2010).

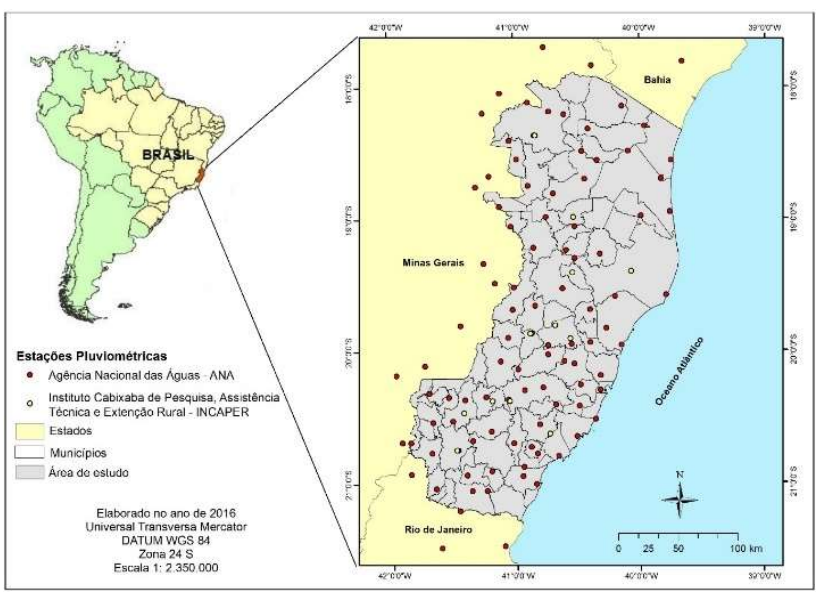

Figura 1. Localização das estações pluviométricas do Espírito Santo e circunvizinhança.

Figure 1. Spatial location of rainfall station within Espírito Santo and neighborhood.

\subsection{Análise estatística}

Com o objetivo de resumir as informações dos dados e descrevê-los em termos numéricos, foi realizada a análise descritiva dos totais precipitados médios mensais e anual. Calcularam-se medidas de tendência central (média e mediana) e medidas de dispersão (desvio padrão e coeficiente de variação). As medidas de dispersão avaliam a homogeneidade dos dados e a dispersão dos mesmos em relação à media. $\mathrm{O}$ coeficiente de variação foi calculado utilizando-se a Equação 1.

$$
\mathrm{Cv}=\frac{\mathrm{s}}{\mu} \cdot 100
$$

em que: $\mathrm{Cv}=$ coeficiente de variação da precipitação mensal ou anual na área em estudo, em \%; S = desvio padrão da precipitação mensal ou anual na área em estudo, em mm; $\mu$ = valor médio da precipitação mensal ou anual na área em estudo, em $\mathrm{mm}$.

Além das medidas de tendência central e de dispersão, foram calculados os coeficientes de curtose $(\mathrm{Ck})$ com o objetivo de mensurar o tipo e grau de assimetria dos dados. $\mathrm{O}$ $\mathrm{Ck}$, que mede o grau de achatamento da amostra, foi calculado conforme a Equação 2.

$$
C_{k}=\frac{n \cdot(n+1)}{(n-1) \cdot(n-2) \cdot(n-3)} \cdot \sum_{i=1}^{n}\left(\frac{X_{i}-\mu}{s}\right)-\frac{3 \cdot(n-1)^{2}}{(n-2) \cdot(n-3)}
$$

em que: $\mathrm{Ck}=$ coeficiente de curtose da precipitação mensal ou anual na área em estudo, adimensional; $\mathrm{n}=$ número de estações da área em estudo, adimensional; $\mathrm{Xi}=$ valor da precipitação mensal ou anual em cada estação, em mm.

Para indicar o grau de distorção da distribuição em relação a uma distribuição simétrica (distribuição normal), onde os dados mais frequentes se concentram mais ao centro em relação aos extremos, foi calculado também o coeficiente de assimetria (Cs), de acordo com a Equação 3.

$C_{s}=\frac{n \cdot(n+1)}{(n-1) \cdot(n-2)} \cdot \sum_{i=1}^{n}\left(\frac{X_{i}-\mu}{s}\right)^{3}$

(Equação 3)

em que: $\mathrm{Cs}=$ coeficiente de assimetria da precipitação mensal ou anual na área em estudo, adimensional.

Para o estabelecimento das equações de regressão polinomial, o processo estatístico desenvolvido consistiu no ajuste de modelos lineares, por meio de regressões polinomiais do primeiro ao quarto grau. Para tanto, tomaram-se variáveis provenientes da combinação das coordenadas geográficas e altitude, as quais foram, posteriormente, selecionadas pelo procedimento Backward de regressão múltipla.

O procedimento Backward permite avaliar todas as variáveis dentro de uma regressão, excluindo automaticamente aquelas que, para o modelo estabelecido, foram não significativas pelo teste $\mathrm{t}$ de Student e não proporcionaram melhorias no coeficiente de determinação, significando que após o procedimento irão permanecer no modelo as variáveis que tenham colaborado com a qualidade final dos ajustes (FERREIRA, 2005).

O software Sigma Plot ${ }^{\circledR}$ foi aplicado para ajuste das regressões polinomias de ordens 1, 2, 3 e 4, cuja estrutura matemática pode ser observada nas Equações 4, 5, 6 e 7, respectivamente. 


$$
\begin{aligned}
& \mathrm{P}_{\mathrm{i}}=\beta_{0}+\beta_{1} \cdot \mathrm{Lo}+\beta_{2} \cdot \mathrm{La}+\beta_{3} \cdot \mathrm{H} \\
& \text { (Equação } \\
& \mathrm{P}_{\mathrm{i}}=\beta_{0}+\beta_{1} \cdot \mathrm{Lo}+\beta_{2} \cdot \mathrm{La}+\beta_{3} \cdot \mathrm{H}+\beta_{4} \cdot \mathrm{Lo}^{2}+\beta_{5} \cdot \mathrm{La}^{2}+ \\
& \beta_{6} \cdot \mathrm{H}^{2}+\beta_{7} \cdot \mathrm{Lo} \cdot \mathrm{La}+\beta_{8} \cdot \mathrm{Lo} \cdot \mathrm{H}+\beta_{9} \cdot \mathrm{La} \cdot \mathrm{H} \\
& \text { (Equação 5) } \\
& \mathrm{P}_{\mathrm{i}}=\beta_{0}+\beta_{1} \cdot \mathrm{Lo}+\beta_{2} \cdot \mathrm{La}+\beta_{3} \cdot \mathrm{H}+\beta_{4} \cdot \mathrm{Lo}^{2}+\beta_{5} \cdot \mathrm{La}^{2}+ \\
& \beta_{6} \cdot \mathrm{H}^{2}+\beta_{7} \cdot \mathrm{Lo}^{3}+\beta_{8} \cdot \mathrm{La}^{3}+\beta_{9} \cdot \mathrm{H}^{3}+\beta_{10} \cdot \mathrm{Lo} \cdot \mathrm{La}+ \\
& \beta_{11} \cdot \mathrm{Lo} \cdot \mathrm{H}+\beta_{12} \cdot \mathrm{La} \cdot \mathrm{H}+\beta_{13} \cdot \mathrm{Lo}^{2} \cdot \mathrm{La}+\beta_{14} \cdot \mathrm{Lo} \cdot \mathrm{La}^{2}+ \\
& \beta_{15} \cdot \mathrm{Lo}^{2} \cdot \mathrm{H}+\beta_{16} \cdot \mathrm{Lo} \cdot \mathrm{H}^{2}+\beta_{17} \cdot \mathrm{La}^{2} \cdot \mathrm{H}+\beta_{18} \cdot \mathrm{La} \cdot \mathrm{H}^{2} \\
& \text { (Equação 6) } \\
& \mathrm{P}_{\mathrm{i}}=\beta_{0}+\beta_{1} \cdot \mathrm{Lo}+\beta_{2} \cdot \mathrm{La}+\beta_{3} \cdot \mathrm{H}+\beta_{4} \cdot \mathrm{Lo}^{2}+\beta_{5} \cdot \mathrm{La}^{2}+ \\
& \beta_{6} \cdot \mathrm{H}^{2}+\beta_{7} \cdot \mathrm{Lo}^{3}+\beta_{8} \cdot \mathrm{La}^{3}+\beta_{9} \cdot \mathrm{H}^{3}+\beta_{10} \cdot \mathrm{Lo}^{4}+ \\
& \beta_{11} \cdot \mathrm{La}^{2}+\beta_{12} \cdot \mathrm{H}^{4}+\beta_{13} \cdot \mathrm{Lo} \cdot \mathrm{La}+\beta_{14} \cdot \mathrm{Lo} \cdot \mathrm{H}+ \\
& \beta_{15} \cdot \mathrm{La} \cdot \mathrm{H}+\beta_{16} \cdot \mathrm{Lo}^{2} \cdot \mathrm{La}+\beta_{17} \cdot \mathrm{Lo}^{2} \cdot \mathrm{H}+\beta_{18} \cdot \mathrm{La}^{2} \cdot \mathrm{H}+ \\
& \beta_{19} \cdot \mathrm{Lo} \cdot \mathrm{La}^{2}+\beta_{20} \cdot \mathrm{Lo} \cdot \mathrm{H}^{2}+\beta_{21} \cdot \mathrm{La} \cdot \mathrm{H}^{2}+ \\
& \beta_{22} \cdot \mathrm{Lo}^{2} \cdot \mathrm{La}^{2}+\beta_{23} \cdot \mathrm{Lo}^{2} \cdot \mathrm{H}^{2}+\beta_{24} \cdot \mathrm{La}^{2} \cdot \mathrm{H}^{2}+ \\
& \beta_{25} \cdot \mathrm{Lo}^{3} \cdot \mathrm{La}+\beta_{26} \cdot \mathrm{Lo}^{3} \cdot \mathrm{H}+\beta_{27} \cdot \mathrm{La}^{3} \cdot \mathrm{H}+\beta_{28} \cdot \mathrm{Lo} \cdot \mathrm{La}^{3}+ \\
& \beta_{29} \cdot \mathrm{Lo} \cdot \mathrm{H}^{3}+\beta_{30} \mathrm{LaH}^{3}
\end{aligned}
$$

em que: $\mathrm{Pi}=$ precipitação média mensal estimada ou anual no local de interesse, em mm; $\mathrm{La}=$ latitude do local de interesse, em graus; Lo $=$ longitude do local de interesse, em graus; $\mathrm{H}=$ altitude do local de interesse, em $\mathrm{mm}$.

A análise de variância (ANOVA) foi calculada com a finalidade de verificar quais dos tipos de regressão polinomial foram significativas nos ajustes aos dados observados, a partir das Soma de Quadrado Total (SQT), Soma de Quadrados devido à Regressão Polinomial (SQRP) e Soma de Quadrados dos Resíduos (SQR). A SQT, SQRP e SQR foram calculadas, respectivamente, a partir das equações 8,9 e 10 .

$$
\begin{aligned}
& \mathrm{SQT}=\mathrm{Y} \cdot \mathrm{Y}^{\prime}-\frac{\left(\sum_{\mathrm{i}=1}^{\mathrm{n}} \mathrm{Y}_{\mathrm{i}}\right)^{2}}{\mathrm{n}} \\
& \mathrm{SQRP}=\mathrm{SQT}-\mathrm{SQR}
\end{aligned}
$$$$
\mathrm{SQR}=\hat{\beta}^{\prime} \cdot \mathrm{X}^{\prime} \cdot \mathrm{Y}-\frac{\left(\sum_{\mathrm{i}=1}^{\mathrm{n}} \mathrm{Y}_{\mathrm{i}}\right)^{2}}{\mathrm{n}}
$$

$\mathrm{SQR}=\hat{\beta^{\prime}} \cdot \mathrm{X}^{\prime} \cdot \mathrm{Y}-\frac{\left(\sum_{\mathrm{i}=1}^{\mathrm{n}} \mathrm{Y}_{\mathrm{i}}\right)^{2}}{\mathrm{n}}$

(Equação 10)

sendo $: \hat{\beta^{\prime}}=\left(X^{\prime} \cdot X\right)^{-1} \cdot X^{\prime} \cdot Y$

(Equação 10)

(Equação 11)

em que: $\mathrm{Y}$ é o vetor $\mathrm{n}$ x 1 dos valores observados da variável $\mathrm{P}_{\mathrm{i}}$; $\mathrm{Y}_{\mathrm{i}}$ são os valores de $P_{i}$ na i-ésima posição; $X$ é a matriz de incidência $n$ $\mathrm{x} \mathrm{p}+1, \beta$ é o vetor de parâmetros desconhecidos $\mathrm{p}+1 \times 1$.

A verificação do ajuste das regressões polinomiais aos dados observados foi realizada testando as seguintes hipóteses: $\mathrm{H}_{0}$ : todos os parâmetros da equação não são significativos (comportamento dos dados não é explicado por meio da regressão).
$\mathrm{H}_{1}$ : existe pelo menos um parâmetro significativo na equação de regressão (regressão significativa).

O coeficiente de determinação $\left(\mathrm{R}^{2}\right)$ foi utilizado para medir o ajuste da superfície e o índice de confiança (c) (CAMARGO; SENTELHAS, 1997) foi utilizado para analisar a precisão e a exatidão dos resultados estimados. Foi calculado o desvio quadrático médio (RMS) a fim de verificar a flutuação estatística dos resíduos gerados pela regressão que melhor se ajustou aos dados, e o erro percentual absoluto médio (EPAM) (SILVA et al., 2011):

$\mathrm{RMS}=\sqrt{\frac{1}{\mathrm{~N}} \cdot \sum_{j=1}^{\mathrm{n}}\left(\mathrm{P}_{\mathrm{o}}-\mathrm{P}_{\mathrm{i}}\right)}$

(Equação 12)

$\operatorname{EPAM}=\frac{1}{N} \cdot \sum_{j=1}^{n} \frac{\left(\left|P_{o}-P_{i}\right|\right)}{P_{o}} 100$

(Equação 13)

em que: $\mathrm{Pi}=$ precipitação média mensal estimada, $\mathrm{Po}=$ precipitação média mensal observada e $\mathrm{N}$ é o número de observações.

\section{RESULTADOS}

Os resultados da análise descritiva das precipitações mensais e anual do Espírito Santo são apresentados na Tabela 1. A Tabela 2 apresenta os valores dos $\mathrm{R}^{2}$, índice c, RMS e EPAM das equações polinomiais ajustadas. Ressalta-se que, de acordo com as análises de variância realizadas, todas as regressões polinomiais ajustadas foram estatisticamente significativas com $5 \%$ de probabilidade, indicando que a variância dos dados estimados pelas regressões ajustadas é menor do que a dos dados originais.

Os modelos de regressão polinomial de ordem 4, ajustados para as precipitações médias mensais e a precipitação média anual, estão apresentados nas Tabela 3 e 4 . Os valores consistem dos coeficientes ajustados para cada variável selecionada pelo procedimento "Backward", com as respectivas significâncias estatísticas pelo teste de $\mathrm{t}$ de Student.

Tabela 1. Estatística descritiva das séries históricas referentes aos totais mensais e anuais da precipitação do Espírito Santo para o período de 1971-2011.

Table 1. Descriptive statistics of the historical series referring to the monthly and annual totals of the precipitation of Espírito Santo for the period 1971-2011.

\begin{tabular}{lcccccc}
\hline Per. & $\begin{array}{c}\text { Média } \\
(\mathrm{mm})\end{array}$ & $\begin{array}{c}\text { Mediana } \\
(\mathrm{mm})\end{array}$ & $\begin{array}{c}\mathrm{S} \\
(\mathrm{mm})\end{array}$ & $\mathrm{C}_{\mathrm{s}}$ & $\mathrm{C}_{\mathrm{k}}$ & $\begin{array}{c}\mathrm{Cv} \\
(\%)\end{array}$ \\
\hline Jan. & 171 & 170 & 34 & 0,33 & 3,14 & 20 \\
Fev. & 100 & 100 & 20 & 0,11 & 2,45 & 20 \\
Mar. & 146 & 140 & 28 & 0,57 & 3,32 & 19 \\
Abr. & 90 & 87 & 25 & 0,57 & 2,91 & 27 \\
Maio & 48 & 44 & 17 & 0,92 & 3,02 & 34 \\
Jun. & 30 & 25 & 13 & 1,01 & 3,47 & 43 \\
Jul. & 38 & 33 & 19 & 0,78 & 3,00 & 51 \\
Ago. & 34 & 31 & 15 & 0,87 & 3,37 & 42 \\
Set. & 58 & 54 & 24 & 0,77 & 3,05 & 41 \\
Out. & 112 & 110 & 20 & 0,57 & 2,98 & 18 \\
Nov. & 200 & 194 & 27 & 0,81 & 3,85 & 13 \\
Dez. & 214 & 213 & 39 & 0,37 & 2,93 & 18 \\
Ano & 1241 & 1210 & 207 & 0,63 & 3,21 & 17 \\
\hline Per.
\end{tabular}

Per. = período; $\mathrm{S}=$ desvio padrão; $\mathrm{Cs}=$ coeficiente de assimetria; $\mathrm{Ck}=$ coeficiente de curtose; $\mathrm{Cv}=$ coeficiente de variação. 
Tabela 2. Coeficientes de determinação $\left(\mathrm{R}^{2}\right)$, índice de confiabilidade (c), desvios quadráticos médios (RMS, em $\mathrm{mm}^{2}$ ) e erros de predição absolutos médios (\%) obtidos pelas regressões polinomiais ajustadas para as precipitações médias históricas mensais e anual do estado do Espírito Santo.

Table 2. Determination coefficient $\left(\mathrm{R}^{2}\right)$, confidence index (c), round mean square error $\left(\mathrm{RMS}\right.$, in $\left.\mathrm{mm}^{2}\right)$ and mean absolute prediction errors (\%) of the polynomial regression adjusted to predict monthly and annual historical average rainfall depth for Espírito Santo.

\begin{tabular}{|c|c|c|c|c|c|c|c|c|c|c|c|c|c|c|c|c|}
\hline \multirow{2}{*}{ Per. } & \multicolumn{4}{|c|}{ Ordem 1} & \multicolumn{4}{|c|}{ Ordem 2} & \multicolumn{4}{|c|}{ Ordem 3} & \multicolumn{4}{|c|}{ Ordem 4} \\
\hline & $\mathrm{R}^{2}$ & $\mathrm{c}$ & RMS & EPAM & $\mathrm{R}^{2}$ & $\mathrm{c}$ & RMS & EPAM & $\mathrm{R}^{2}$ & $\mathrm{c}$ & RMS & EPAM & $\mathrm{R}^{2}$ & c & RMS & EPAM \\
\hline Jan. & 0,5 & 0,6 & 24,1 & 11,89 & 0,7 & 0,7 & 19,6 & 9,3 & 0,8 & 0,8 & 16,5 & 7,5 & 0,8 & 0,9 & 13,5 & 6,5 \\
\hline Fev. & 0,2 & 0,3 & 18,7 & 15,5 & 0,5 & 0,6 & 14,0 & 11,9 & 0,6 & 0,7 & 11,9 & 9,8 & 0,7 & 0,8 & 10,7 & 8,8 \\
\hline Mar. & 0,3 & 0,4 & 22,8 & 12,4 & 0,5 & 0,6 & 19,4 & 10,7 & 0,7 & 0,7 & 16,1 & 8,4 & 0,8 & 0,8 & 14,1 & 7,6 \\
\hline Abr. & 0,4 & 0,4 & 19,5 & 18,7 & 0,6 & 0,6 & 16,1 & 13,9 & 0,7 & 0,8 & 13,4 & 11,6 & 0,8 & 0,9 & 10,8 & 9,4 \\
\hline Mai. & 0,5 & 0,6 & 11,4 & 19,4 & 0,7 & 0,8 & 9,1 & 13,9 & 0,8 & 0,8 & 7,4 & 12,2 & 0,9 & 0,9 & 6,3 & 10,7 \\
\hline Jun. & 0,6 & 0,7 & 7,9 & 23,9 & 0,8 & 0,8 & 5,8 & 13,9 & 0,9 & 0,9 & 4,8 & 13,1 & 0,9 & 0,9 & 4,1 & 11,4 \\
\hline Jul. & 0,6 & 0,7 & 12,3 & 35,6 & 0,8 & 0,8 & 9,1 & 20,5 & 0,8 & 0,9 & 8,2 & 20,0 & 0,9 & 0,9 & 6,9 & 16,6 \\
\hline Ago. & 0,6 & 0,6 & 9,7 & 25,6 & 0,7 & 0,8 & 8,0 & 18,3 & 0,8 & 0,8 & 7,2 & 17,4 & 0,9 & 0,9 & 5,7 & 14,5 \\
\hline Set. & 0,6 & 0,6 & 15,9 & 24,6 & 0,7 & 0,8 & 12,9 & 16,3 & 0,8 & 0,8 & 11,6 & 15,9 & 0,8 & 0,9 & 9,9 & 14,0 \\
\hline Out. & 0,4 & 0,5 & 15,1 & 10,4 & 0,5 & 0,6 & 13,6 & 8,8 & 0,7 & 0,7 & 11,6 & 7,5 & 0,7 & 0,8 & 10,5 & 7,1 \\
\hline Nov. & 0,2 & 0,3 & 23,3 & 8,8 & 0,3 & 0,4 & 21,9 & 8,3 & 0,5 & 0,6 & 18,7 & 7,3 & 0,7 & 0,7 & 15,9 & 5,9 \\
\hline Dez. & 0,5 & 0,6 & 27,2 & 10,2 & 0,6 & 0,7 & 23,4 & 0,7 & 0,7 & 0,8 & 19,6 & 7,1 & 0,8 & 0,8 & 17,2 & 6,5 \\
\hline Ano & 0,5 & 0,5 & 150,0 & 9,4 & 0,5 & 0,6 & 144,9 & 0,6 & 0,6 & 0,7 & 125,4 & 7,8 & 0,7 & 0,8 & 110,0 & 6,9 \\
\hline
\end{tabular}

Per. $=$ período.

Tabela 3. Modelos para estimar as precipitações mensais (Janeiro a Julho) para o estado do Espírito Santo. Table 3. Models for estimation of monthly (January to July) precipitations for the of Espírito Santo state.

\begin{tabular}{|c|c|c|c|c|c|c|c|}
\hline \multirow{2}{*}{ Variável } & \multicolumn{7}{|c|}{ Meses } \\
\hline & Janeiro & Fevereiro & Março & Abril & Maio & Junho & Julho \\
\hline$\beta_{0}$ (Intercepto) & $1373497,33^{\mathrm{ns}}$ & $19790696,87^{*}$ & $1736810,61^{\mathrm{ns}}$ & $312748,14^{\mathrm{ns}}$ & $645912,73^{\text {ns }}$ & $445660,22^{\text {ns }}$ & $-927854,63^{\mathrm{ns}}$ \\
\hline$\beta_{1}(\mathrm{Lo})$ & $-107406,07^{\mathrm{ns}}$ & $1264776,88^{*}$ & $2033,01^{\mathrm{ns}}$ & $190090,02^{\mathrm{ns}}$ & $27759,19^{\mathrm{ns}}$ & $-473,04^{\mathrm{ns}}$ & $-13361,70^{\mathrm{ns}}$ \\
\hline$\beta_{2}(\mathrm{La})$ & $571672,03^{\mathrm{ns}}$ & $1510095,65^{*}$ & $177356,49^{\mathrm{ns}}$ & $-369157,53^{\mathrm{ns}}$ & $131,21^{\mathrm{ns}}$ & $100632,86^{\mathrm{ns}}$ & $-118398,76^{* *}$ \\
\hline$\beta_{3}(\mathrm{H})$ & $14656,04^{* *}$ & $9260,41^{*}$ & $7660,22^{\mathrm{ns}}$ & $6759,92^{\mathrm{ns}}$ & $2253,23^{\mathrm{ns}}$ & $4531,19^{\mathrm{ns}}$ & $9408,24^{\mathrm{ns}}$ \\
\hline$\beta_{4}\left(\mathrm{Lo}^{2}\right)$ & $-13399,05^{\mathrm{ns}}$ & $24010,75^{*}$ & $-4967,24^{\mathrm{ns}}$ & $6090,40^{\mathrm{ns}}$ & $-217,46^{\mathrm{ns}}$ & $-91,19^{\mathrm{ns}}$ & $670,81^{\mathrm{ns}}$ \\
\hline$\beta_{5}\left(\mathrm{La}^{2}\right)$ & $-1771,50^{\mathrm{ns}}$ & $11061,41^{\mathrm{ns}}$ & $8711,25^{\mathrm{ns}}$ & $-30405,57^{\mathrm{ns}}$ & $70,58^{\mathrm{ns}}$ & $6004,24^{\mathrm{ns}}$ & $-8557,63^{\mathrm{ns}}$ \\
\hline$\beta_{6}\left(\mathrm{H}^{2}\right)$ & $-0,30^{\mathrm{ns}}$ & $0,46^{\mathrm{ns}}$ & $-0,56^{\mathrm{ns}}$ & $0,77^{\mathrm{ns}}$ & $0,41^{\mathrm{ns}}$ & $0,52^{\mathrm{ns}}$ & $1,02^{* *}$ \\
\hline$\beta_{7}\left(\mathrm{Lo}^{3}\right)$ & $-469,85^{\mathrm{ns}}$ & $27,25^{\mathrm{ns}}$ & $-126,41^{\mathrm{ns}}$ & $18,33^{\text {ns }}$ & $3,14^{\mathrm{ns}}$ & $21,02^{\mathrm{ns}}$ & $17,86^{\mathrm{ns}}$ \\
\hline$\beta_{8}\left(\mathrm{La}^{3}\right)$ & $870,72^{\mathrm{ns}}$ & $360,42^{* *}$ & $-138,71^{\mathrm{ns}}$ & $-422,61^{\mathrm{ns}}$ & $-260,94^{\mathrm{ns}}$ & $3,61^{\mathrm{ns}}$ & $-152,90^{\mathrm{ns}}$ \\
\hline$\beta_{9}\left(\mathrm{H}^{3}\right)$ & $-4,29 \cdot 10^{-5 \mathrm{~ns}}$ & $-9,50 \cdot 10^{-7 \mathrm{~ns}}$ & $-3,02 \cdot 10^{-7 \mathrm{~ns}}$ & $1,93 \cdot 10^{-5 \mathrm{~ns}}$ & $1,26 \cdot 10^{-5 \mathrm{~ns}}$ & $1,47 \cdot 10^{-5 \mathrm{~ns}}$ & $3,07 \cdot 10^{-5 \mathrm{~ns}}$ \\
\hline$\beta_{10}\left(\mathrm{Lo}^{4}\right)$ & $-5,96^{\mathrm{ns}}$ & $-2,45^{*}$ & $-1,25^{\mathrm{ns}}$ & $-0,94^{\mathrm{ns}}$ & $0,17^{\mathrm{ns}}$ & $0,28^{\mathrm{ns}}$ & $-8,1 \cdot 10^{-3 \mathrm{~ns}}$ \\
\hline$\beta_{11}\left(\mathrm{La}^{4}\right)$ & $-7,93^{\mathrm{ns}}$ & $-3,29^{\mathrm{ns}}$ & $-10,44^{* *}$ & $-8,07^{* *}$ & $-4,16^{* *}$ & $-2,30^{* *}$ & $-3,50^{\mathrm{ns}}$ \\
\hline$\beta_{12}\left(\mathrm{H}^{4}\right)$ & $2,51 \cdot 10^{-11 \mathrm{~ns}}$ & $2,57 \cdot 10^{-10 \mathrm{~ns}}$ & $-4,71 \cdot 10^{-10 \mathrm{~ns}}$ & $4,43 \cdot 10^{-10 \mathrm{~ns}}$ & $5,70 \cdot 10^{-10^{* *}}$ & $2,31 \cdot 10^{-10 \mathrm{~ns}}$ & $5,81 \cdot 10^{-10 \mathrm{~ns}}$ \\
\hline$\beta_{13}$ (Lo La) & $43877,61^{\mathrm{ns}}$ & $100763,78^{*}$ & $6342,42^{\mathrm{ns}}$ & $1633,59^{\mathrm{ns}}$ & $-134,40^{\mathrm{ns}}$ & $1331,17^{\mathrm{ns}}$ & $-977,89^{\mathrm{ns}}$ \\
\hline$\beta_{14}(\mathrm{Lo} \mathrm{H})$ & $1024,34^{* *}$ & $661,03^{*}$ & $509,49^{\mathrm{ns}}$ & $508,7184^{\mathrm{ns}}$ & $168,30^{\mathrm{ns}}$ & $337,84^{\mathrm{ns}}$ & $706,81^{* *}$ \\
\hline$\beta_{15}(\mathrm{La} \mathrm{H})$ & $63,46^{* *}$ & $40,68^{*}$ & $64,31^{\mathrm{ns}}$ & $-10,18^{\mathrm{ns}}$ & $-0,66^{\mathrm{ns}}$ & $-1,74^{\mathrm{ns}}$ & $-13,86^{\mathrm{ns}}$ \\
\hline$\beta_{16}\left(\mathrm{Lo}^{2} \mathrm{La}\right)$ & $1685,93^{\mathrm{ns}}$ & $2450,86^{*}$ & $-117,76^{\mathrm{ns}}$ & $464,94^{\mathrm{ns}}$ & $-174,15^{\mathrm{ns}}$ & $-108,22^{\mathrm{ns}}$ & $65,82^{\mathrm{ns}}$ \\
\hline$\beta_{17}\left(\mathrm{Lo}^{2} \mathrm{H}\right)$ & $24,56^{\mathrm{ns}}$ & $16,21^{*}$ & $11,92^{\mathrm{ns}}$ & $12,66^{\mathrm{ns}}$ & $4,20^{\mathrm{ns}}$ & $8,39^{\text {ns }}$ & $17,55^{* *}$ \\
\hline$\beta_{18}\left(\mathrm{La}^{2} \mathrm{H}\right)$ & $3,13^{* *}$ & $1,98^{*}$ & $3,30^{\mathrm{ns}}$ & $-0,63^{\mathrm{ns}}$ & $-0,09^{\mathrm{ns}}$ & $-0,13^{\mathrm{ns}}$ & $-0,77^{\mathrm{ns}}$ \\
\hline$\beta_{19}\left(\mathrm{Lo} \mathrm{La}^{2}\right)$ & $-1303,01^{\mathrm{ns}}$ & $39,80^{\mathrm{ns}}$ & $640,56^{\mathrm{ns}}$ & $-894,07^{\mathrm{ns}}$ & $365,42^{\mathrm{ns}}$ & $283,12^{\mathrm{ns}}$ & $-206,05^{\mathrm{ns}}$ \\
\hline$\beta_{20}\left(\mathrm{Lo} \mathrm{H}^{2}\right)$ & $-0,01^{\mathrm{ns}}$ & $0,02^{\mathrm{ns}}$ & $-0,03^{\mathrm{ns}}$ & $0,04^{\mathrm{ns}}$ & $0,02^{\mathrm{ns}}$ & $0,03^{\mathrm{ns}}$ & $0,05^{* *}$ \\
\hline$\beta_{21}\left(\mathrm{La} \mathrm{H}^{2}\right)$ & $-0,01^{\mathrm{ns}}$ & $-5,1 \cdot 10^{-3 \mathrm{~ns}}$ & $1,9 \cdot 10^{-3 \mathrm{~ns}}$ & $-4,8 \cdot 10^{-3 \mathrm{~ns}}$ & $-3,3 \cdot 10^{-3 \mathrm{~ns}}$ & $-2,3 \cdot 10^{-3 \mathrm{~ns}}$ & $-3,9 \cdot 10^{-3 \mathrm{~ns}}$ \\
\hline$\beta_{22}\left(\mathrm{Lo}^{2} \mathrm{La}^{2}\right)$ & $-41,80^{* *}$ & $-10,15^{\mathrm{ns}}$ & $-3,80^{\mathrm{ns}}$ & $-14,62^{\mathrm{ns}}$ & $3,22^{\mathrm{ns}}$ & $0,18^{\mathrm{ns}}$ & $-4,70^{\mathrm{ns}}$ \\
\hline$\beta_{23}\left(\mathrm{Lo}^{2} \mathrm{H}^{2}\right)$ & $-2 \cdot 10^{-4 n s}$ & $3 \cdot 10^{-4 \mathrm{~ns}}$ & $-4 \cdot 10^{-4 n s}$ & $5 \cdot 10^{-4 \mathrm{~ns}}$ & $3 \cdot 10^{-3 \mathrm{~ns}}$ & $3 \cdot 10^{-4 \mathrm{~ns}}$ & $7 \cdot 10^{-4 * *}$ \\
\hline$\beta_{24}\left(\mathrm{La}^{2} \mathrm{H}^{2}\right)$ & $-2 \cdot 10^{-4 \mathrm{~ns}}$ & $-1 \cdot 10^{-4 n s}$ & $3,89 \cdot 10^{-5 \mathrm{~ns}}$ & $-1 \cdot 10^{-4 n s}$ & $-1 \cdot 10^{-3 \mathrm{~ns}}$ & $-5,89 \cdot 10^{-5 \mathrm{~ns}}$ & $-1 \cdot 10^{-4 \mathrm{~ns}}$ \\
\hline$\beta_{25}\left(\mathrm{Lo}^{3} \mathrm{La}\right)$ & $26,88^{* *}$ & $23,20^{*}$ & $0,45^{\mathrm{ns}}$ & $8,46^{\mathrm{ns}}$ & $-2,38^{\mathrm{ns}}$ & $-0,97^{\mathrm{ns}}$ & $1,98^{\mathrm{ns}}$ \\
\hline$\beta_{26}\left(\mathrm{Lo}^{3} \mathrm{H}\right)$ & $0,20^{\mathrm{ns}}$ & $0,13^{*}$ & $0,09^{\mathrm{ns}}$ & $0,11^{\mathrm{ns}}$ & $0,03^{\mathrm{ns}}$ & $0,07^{\mathrm{ns}}$ & $0,15^{* *}$ \\
\hline$\beta_{27}\left(\mathrm{La}^{3} \mathrm{H}\right)$ & $0,05^{* *}$ & $0,03^{*}$ & $0,06^{\mathrm{ns}}$ & $-0,01^{\mathrm{ns}}$ & $-2,5 \cdot 10^{-3 \mathrm{~ns}}$ & $-2,9 \cdot 10^{-3 \mathrm{~ns}}$ & $-0,01^{\mathrm{ns}}$ \\
\hline$\beta_{28}\left(\mathrm{Lo} \mathrm{La}^{3}\right)$ & $36,54^{* *}$ & $15,10^{*}$ & $16,54^{\mathrm{ns}}$ & $5,02^{\mathrm{ns}}$ & $1,56^{\mathrm{ns}}$ & $4,48^{\mathrm{ns}}$ & $2,95^{\mathrm{ns}}$ \\
\hline$\beta_{29}\left(\mathrm{Lo} \mathrm{H}^{3}\right)$ & $-1,08 \cdot 10^{-6 \mathrm{~ns}}$ & $-4,58 \cdot 10^{-8^{*}}$ & $-6,81 \cdot 10^{-6 n s}$ & $5,06 \cdot 10^{-7 \mathrm{~ns}}$ & $3,22 \cdot 10^{-7 \mathrm{~ns}}$ & $3,76 \cdot 10^{-7 \mathrm{~ns}}$ & $7,88 \cdot 10^{-7 \mathrm{~ns}}$ \\
\hline$\beta_{30}\left(\mathrm{La} \mathrm{H}^{3}\right)$ & $5,71 \cdot 10^{-8 \mathrm{~ns}}$ & $5,43 \cdot 10^{-8^{*}}$ & $-1,75 \cdot 10^{-7 \mathrm{~ns}}$ & $-3,25 \cdot 10^{-8 \mathrm{~ns}}$ & $2,77 \cdot 10^{-8 \mathrm{~ns}}$ & $-1,83 \cdot 10^{-8 \mathrm{~ns}}$ & $-3,21 \cdot 10^{-8 n s}$ \\
\hline
\end{tabular}

* - significativo a $1 \%$ pelo teste $\mathrm{t}$ de Student; ** - significativo a $5 \%$ pelo teste t de Student; ns - não significativo.

\section{DISCUSSÃO}

Verifica-se, na Tabela 1, que as maiores precipitações se dão de outubro a março, com valores variando de $100 \mathrm{~mm}$ a aproximadamente $214 \mathrm{~mm}$. As menores precipitações ocorrem entre abril e setembro, com valores variando de 30 a $90 \mathrm{~mm}$.
Tal comportamento reflete a classificação de Köppen predominante para a região, com inverno seco e verão chuvoso (Cwa) (ALVARES et al., 2013).

Analisando a variabilidade dos dados com base nos coeficientes de variação $(\mathrm{Cv})$ observa-se que, de acordo com a 
classificação de Pimentel-Gomes (1985), as precipitações apresentaram variação média $(\mathrm{Cv}$ entre 10 e $20 \%)$, alta $(\mathrm{Cv}$ entre 20 e $30 \%)$ e muito alta $(\mathrm{Cv}>30 \%)$. Isto indica que há grande variabilidade de precipitação no estado do Espírito Santo, corroborando com os resultados encontrados em outros trabalhos (MELLO et al., 2012; SILVA et al., 2011; ULIANA et al., 2013), nos quais foram identificadas regiões pluviométricas distintas no estado.

O período seco (maio a agosto) se destaca por apresentar as maiores variabilidades, de acordo com o Cv. Para setembro, por exemplo, o comportamento é explicado por se tratar de um mês caracterizado pela mudança entre o período seco e período chuvoso, o qual foi verificado, na série histórica, situações nas quais o período chuvoso se iniciou com atraso, gerando precipitações mensais quase nulas, ou nulas em alguns anos. Tal variabilidade pode ser explicada, em parte por diferenças no início e na duração do período chuvoso em diferentes localidades do Espírito Santo. Fenômenos periódicos de escala global de circulação como o El niño e La niña (periodicidade de aproximadamente 4 anos) e fenômenos relacionados ao ciclo solar (periodicidade de aproximadamente 11 anos) também influenciam no início do período chuvoso e, consequentemente, no total precipitado nos meses de transição do período seco ara o chuvoso (MINUZZI et al., 2006).

Tabela 4. Modelos para estimar as precipitações mensais (Agosto a Dezembro) e anuais para o estado do Espírito Santo.

Table 4. Models for estimation of monthly (August to December) and annual precipitations for the of Espírito Santo state.

\begin{tabular}{|c|c|c|c|c|c|c|}
\hline \multirow{2}{*}{ Variável } & \multicolumn{5}{|c|}{ Meses } & \multirow{2}{*}{ Anual } \\
\hline & Agosto & Setembro & Outubro & Novembro & Dezembro & \\
\hline$\beta_{0}$ (Intercepto) & $3357812,06^{\mathrm{ns}}$ & $-2669649,35^{\mathrm{ns}}$ & $-1276626,50^{\mathrm{ns}}$ & $-687094,71^{\mathrm{ns}}$ & $644419,63^{\mathrm{ns}}$ & $11109141,36^{\mathrm{ns}}$ \\
\hline$\beta_{1}(\mathrm{Lo})$ & $104806,77^{\mathrm{ns}}$ & $-80786,33^{\mathrm{ns}}$ & $-97144,75^{\mathrm{ns}}$ & $-36893,55^{\mathrm{ns}}$ & $60019,80^{\mathrm{ns}}$ & $555142,37^{\mathrm{ns}}$ \\
\hline$\beta_{2}(\mathrm{La})$ & $546731,53^{* *}$ & $-268612,04^{\mathrm{ns}}$ & $-52156,95^{\mathrm{ns}}$ & $34499,87^{\mathrm{ns}}$ & $99909,10^{\mathrm{ns}}$ & $505646,40^{\mathrm{ns}}$ \\
\hline$\beta_{3}(\mathrm{H})$ & $10522,62^{*}$ & $14070,28^{* *}$ & $10075,19^{\mathrm{ns}}$ & $21469,59^{* *}$ & $5540,40^{\mathrm{ns}}$ & $54106,73^{\mathrm{ns}}$ \\
\hline$\beta_{4}\left(\mathrm{Lo}^{2}\right)$ & $-11028,96^{\mathrm{ns}}$ & $-135,48^{\mathrm{ns}}$ & $-1675,90^{\mathrm{ns}}$ & $-11592,04^{\mathrm{ns}}$ & $2772,35^{\mathrm{ns}}$ & $5366,45^{\mathrm{ns}}$ \\
\hline$\beta_{5}\left(\mathrm{La}^{2}\right)$ & $-30098,48^{*}$ & $-19031,26^{\mathrm{ns}}$ & $1520,05^{\mathrm{ns}}$ & $-52606,03^{\mathrm{ns}}$ & $1112,58^{\mathrm{ns}}$ & $22747,59^{\mathrm{ns}}$ \\
\hline$\beta_{6}\left(\mathrm{H}^{2}\right)$ & $0,84^{*}$ & $1,43^{* *}$ & $0,90^{\mathrm{ns}}$ & $1,36^{\mathrm{ns}}$ & $-0,08^{\mathrm{ns}}$ & $-0,21^{\mathrm{ns}}$ \\
\hline$\beta_{7}\left(\mathrm{Lo}^{3}\right)$ & $-514,17^{*}$ & $12,93^{\mathrm{ns}}$ & $-2,19^{\mathrm{ns}}$ & $-610,15^{\mathrm{ns}}$ & $-5,17^{\mathrm{ns}}$ & $-61,85^{\mathrm{ns}}$ \\
\hline$\beta_{8}\left(\mathrm{La}^{3}\right)$ & $-165,43^{* *}$ & $-299,50^{\mathrm{ns}}$ & $197,68^{\mathrm{ns}}$ & $669,99^{\mathrm{ns}}$ & $688,23^{\mathrm{ns}}$ & $0,45^{\mathrm{ns}}$ \\
\hline$\beta_{9}\left(\mathrm{H}^{3}\right)$ & $2,34 \cdot 10^{-5^{*}}$ & $4,06 \cdot 10^{-5 \mathrm{~ns}}$ & $1,70 \cdot 10^{-5 \mathrm{~ns}}$ & $2,03 \cdot 10^{-5 \mathrm{~ns}}$ & $-4,06 \cdot 10^{-5 n s}$ & $-1 \cdot 10^{-4 \mathrm{~ns}}$ \\
\hline$\beta_{10}\left(\mathrm{Lo}^{4}\right)$ & $-5,84^{*}$ & $2,4 \cdot 10^{-3 \mathrm{~ns}}$ & $-0,17^{\mathrm{ns}}$ & $-8,68^{\mathrm{ns}}$ & $-1,34^{\mathrm{ns}}$ & $-3,83^{\mathrm{ns}}$ \\
\hline$\beta_{11}\left(\mathrm{La}^{4}\right)$ & $-3,72^{*}$ & $-3,19^{\mathrm{ns}}$ & $-3,02^{\mathrm{ns}}$ & $-7,95^{\mathrm{ns}}$ & $-4,12^{\mathrm{ns}}$ & $-63,20^{* *}$ \\
\hline$\beta_{12}\left(\mathrm{H}^{4}\right)$ & $5,38 \cdot 10^{-10^{*}}$ & $6,35 \cdot 10^{-5 \mathrm{~ns}}$ & $3,08 \cdot 10^{-10 \mathrm{~ns}}$ & $5,21 \cdot 10^{-10 \mathrm{~ns}}$ & $7,89 \cdot 10^{-11 \mathrm{~ns}}$ & $2,50 \cdot 10^{-9 \mathrm{~ns}}$ \\
\hline$\beta_{13}(\mathrm{Lo} \mathrm{La})$ & $68549,77^{*}$ & $-2536,10^{\mathrm{ns}}$ & $-6367,44^{\mathrm{ns}}$ & $51495,20^{\mathrm{ns}}$ & $5331,09^{\mathrm{ns}}$ & $16210,33^{\mathrm{ns}}$ \\
\hline$\beta_{14}(\mathrm{Lo} \mathrm{H})$ & $784,69^{*}$ & $1051,94^{* *}$ & $731,46^{\mathrm{ns}}$ & $1574,10^{* *}$ & $393,48^{\mathrm{ns}}$ & $3774,18^{\mathrm{ns}}$ \\
\hline$\beta_{15}(\mathrm{La} \mathrm{H})$ & $-10,24^{*}$ & $-9,65^{\mathrm{ns}}$ & $26,18^{\mathrm{ns}}$ & $9,98^{\mathrm{ns}}$ & $8,53^{\mathrm{ns}}$ & $200,06^{\mathrm{ns}}$ \\
\hline$\beta_{16}\left(\mathrm{Lo}^{2} \mathrm{La}\right)$ & $2263,83^{*}$ & $160,97^{\mathrm{ns}}$ & $-89,28^{\mathrm{ns}}$ & $2900,23^{\mathrm{ns}}$ & $535,31^{\mathrm{ns}}$ & $-203,87^{\mathrm{ns}}$ \\
\hline$\beta_{17}\left(\mathrm{Lo}^{2} \mathrm{H}\right)$ & $19,40^{*}$ & $26,12^{* *}$ & $18,03^{\mathrm{ns}}$ & $38,63^{* *}$ & $9,44^{\mathrm{ns}}$ & $90,03^{\mathrm{ns}}$ \\
\hline$\beta_{18}\left(\mathrm{La}^{2} \mathrm{H}\right)$ & $-0,57^{*}$ & $-0,58^{\mathrm{ns}}$ & $1,22^{\mathrm{ns}}$ & $0,31^{\mathrm{ns}}$ & $0,23^{\mathrm{ns}}$ & $9,35^{\mathrm{ns}}$ \\
\hline$\beta_{19}\left(\mathrm{Lo} \mathrm{La}^{2}\right)$ & $-1240,83^{*}$ & $-510,45^{\mathrm{ns}}$ & $-202,44^{\mathrm{ns}}$ & $-3518,36^{\mathrm{ns}}$ & $-914,34^{\mathrm{ns}}$ & $1203,37^{\mathrm{ns}}$ \\
\hline$\beta_{20}\left(\mathrm{Lo} \mathrm{H}^{2}\right)$ & $0,04^{*}$ & $0,07^{* *}$ & $0,05^{\mathrm{ns}}$ & $0,07^{\mathrm{ns}}$ & $1 \cdot 10^{-3 \mathrm{~ns}}$ & $0,01^{\mathrm{ns}}$ \\
\hline$\beta_{21}\left(\mathrm{La} \mathrm{H}^{2}\right)$ & $-2,4 \cdot 10^{-3 \mathrm{~ns}}$ & $-4,4 \cdot 10^{-3 \mathrm{~ns}}$ & $-6,1 \cdot 10^{-3 \mathrm{~ns}}$ & $-0,01^{\mathrm{ns}}$ & $-0,01^{* *}$ & $-0,05^{\mathrm{ns}}$ \\
\hline$\beta_{22}\left(\mathrm{Lo}^{2} \mathrm{La}^{2}\right)$ & $-17,44^{*}$ & $-5,52^{\mathrm{ns}}$ & $-9,94^{\mathrm{ns}}$ & $-65,30^{\mathrm{ns}}$ & $-28,44^{\mathrm{ns}}$ & $-70,31^{\mathrm{ns}}$ \\
\hline$\beta_{23}\left(\mathrm{Lo}^{2} \mathrm{H}^{2}\right)$ & $5 \cdot 10^{-4 n s} *$ & $9 \cdot 10^{-4 \mathrm{~ns}^{* *}}$ & $6 \cdot 10^{-4 \mathrm{~ns}}$ & $9 \cdot 10^{-4 \mathrm{~ns}}$ & $-4,39 \cdot 10^{-6 n s}$ & $6,18 \cdot 10^{-5 \mathrm{~ns}}$ \\
\hline$\beta_{24}\left(\mathrm{La}^{2} \mathrm{H}^{2}\right)$ & $-6,28 \cdot 10^{-5 n s}$ & $-1 \cdot 10^{-4 n s}$ & $-2 \cdot 10^{-4 n s}$ & $-3 \cdot 10^{-4 n s}$ & $-3 \cdot 10^{-4^{* *}}$ & $-1,3 \cdot 10^{-3 \mathrm{~ns}}$ \\
\hline$\beta_{25}\left(\mathrm{Lo}^{3} \mathrm{La}\right)$ & $23,98^{*}$ & $2,93^{\mathrm{ns}}$ & $2,15^{\mathrm{ns}}$ & $44,02^{\mathrm{ns}}$ & $13,06^{\mathrm{ns}}$ & $20,05^{\mathrm{ns}}$ \\
\hline$\beta_{26}\left(\mathrm{Lo}^{3} \mathrm{H}\right)$ & $0,16^{*}$ & $0,22^{* *}$ & $0,15^{\mathrm{ns}}$ & $0,32^{* *}$ & $0,08^{\text {ns }}$ & $0,72^{\mathrm{ns}}$ \\
\hline$\beta_{27}\left(\mathrm{La}^{3} \mathrm{H}\right)$ & $-0,01^{*}$ & $-0,01^{\mathrm{ns}}$ & $0,02^{\mathrm{ns}}$ & $1,7 \cdot 10^{-3 \mathrm{~ns}}$ & $4 \cdot 10^{-3 \mathrm{~ns}}$ & $0,14^{\mathrm{ns}}$ \\
\hline$\beta_{28}\left(\mathrm{Lo} \mathrm{La}^{3}\right)$ & $3,07^{*}$ & $-1,19^{\mathrm{ns}}$ & $10,57^{\mathrm{ns}}$ & $31,46^{\mathrm{ns}}$ & $24,54^{\mathrm{ns}}$ & $120,54^{\mathrm{ns}}$ \\
\hline$\beta_{29}\left(\mathrm{Lo} \mathrm{H}^{3}\right)$ & $6,02 \cdot 10^{-7^{*}}$ & $1,10 \cdot 10^{-6 \mathrm{~ns}}$ & $4,03 \cdot 10^{-7 \mathrm{~ns}}$ & $5,03 \cdot 10^{-7 \mathrm{~ns}}$ & $-1,05 \cdot 10^{-6 \mathrm{~ns}}$ & $-2,92 \cdot 10^{-6 \mathrm{~ns}}$ \\
\hline$\beta_{30}\left(\mathrm{La} \mathrm{H}^{3}\right)$ & $-1,65 \cdot 10^{-8^{*}}$ & $1,70 \cdot 10^{-7 \mathrm{~ns}}$ & $3,93 \cdot 10^{-8 \mathrm{~ns}}$ & $1,38 \cdot 10^{-8 n s}$ & $9,57 \cdot 10^{-8 \mathrm{~ns}}$ & $-6,03 \cdot 10^{-7 \mathrm{~ns}}$ \\
\hline
\end{tabular}

* - significativo a $1 \%$ pelo teste t de Student; ** - significativo a 5\% pelo teste t de Student; ns - não significativo.

No período seco ocorre variabilidade tanto temporal quanto espacial, principalmente esta última, provocada por diferentes características climáticas dentro do estado do Espírito Santo (MELLO et al., 2012; Silva et al., 2011; ULIANA et al., 2013). Nesses períodos a precipitação apresenta valores baixos (em média $42 \mathrm{~mm}$ ), sendo que uma alteração nesta, por menor que seja, pode refletir em maiores coeficientes de variação.

A precipitação é maior em regiões onde a altitude é mais elevada, quando comparada com as de regiões de menor altitude (SANT'ANNA NETO, 2005; SILVA et al., 2011; ULIANA et al., 2013), demonstrando que independentemente das características tropicais presentes no estado, a altitude torna-se um importante fator de controle climático, impondose ao aspecto latitudinal.

Finalmente, ainda com relação à Tabela 1, todos os valores médios de precipitação se mostraram maiores que as medianas, determinando a tendência de agrupamento dos dados à direita, em relação à curva de distribuição normal, confirmada pelos coeficientes de assimetria (Cs), que foram todos positivos.

Os coeficientes de curtose (Ck) também se apresentaram positivos, indicando que a distribuição dos dados é do tipo leptocúrtica, ou seja, é mais afilada que a distribuição normal, sendo relativamente mais fácil se obter valores que se aproximem da média e vários múltiplos do desvio padrão. 
De acordo com os coeficientes de determinação $\left(\mathrm{R}^{2}\right)$ apresentados na Tabela 2, a regressão que melhor se ajustou aos dados foi a de quarta ordem, explicando até $90 \%$ das variabilidades ocorridas nos índices de precipitação. O mês de novembro apresentou o menor $\mathrm{R}^{2}$ para todas as regressões ajustadas, o que se deve à maior variabilidade espacial da precipitação. Nesse mês, as porções Norte e Noroeste ainda se encontram no período seco (atraso no início do período chuvoso), enquanto as demais já estão no período chuvoso (SILVA et al., 2011), configurando regiões de características pluviométricas distintas (KELLER FILHO et al., 2005; MELO JÚNIOR et al., 2006), em especial com relação ao início da estação chuvosa (MINUZZI et al., 2007).

O RMS apresentado na Tabela 2 foi empregado a fim de medir o quanto o modelo errou ao ajustar a precipitação. Os menores valores de RMS foram os das regressões de quarta ordem (Tabela 2), o que mostra que há menor variação entre os dados observados e estimados pela regressão polinomial de quarta ordem, quando comparado às demais. Conforme os resultados, os meses de maio a agosto foram os que apresentaram as menores diferenças de precipitação entre os valores observados e estimados, isto é, as regressões de quarta ordem foram mais precisas nestes meses. Os valores de EPAM e do índice c confirmam o melhor desempenho das regressões de quarta ordem, as quais proporcionaram os menores erros e maior precisão e exatidão para todos os meses.

De maneira geral, observa-se que os modelos de maior ordem se ajustaram melhor aos dados observados do que aqueles de menor ordem, de forma semelhante ao apresentado em trabalho desenvolvido para o estado da Paraíba (GOMES et al., 2015). A Figura 2 mostra as curvas das regressões polinomiais em relação aos dados observados para os meses os quais os ajustes apresentaram melhor desempenho (junho), pior desempenho (novembro) e desempenhos intermediários (janeiro e março). O melhor desempenho da regressão polinomial de quarta ordem fica evidente, especialmente em estações onde as médias de precipitação mensal foram mais altas ou mais baixas.

O mês de novembro foi o de pior desempenho da regressão de $4^{\mathrm{a}}$ ordem. Para este mês, deve-se realizar um novo estudo onde possam ser inseridas variáveis que influenciam diretamente na precipitação ou mesmo realizar a divisão do estado em regiões de altitude homogêneas e efetuar o ajuste dos modelos estatísticos para estas regiões.

Dessa forma fica evidenciado que o modelo de regressão polinomial de $4^{\mathrm{a}}$ ordem é uma importante ferramenta para estimar dados de precipitações mensais e precipitação anual para estado do Espírito Santo, podendo ser utilizado para fazer estimativas para locais com precipitação desconhecida dentro da região estudada.

Verifica-se, nas Tabelas 3 e 4, que vários modelos apresentam coeficientes de ajuste $(\beta)$ não significativos, enquanto que o mês de outubro não apresentou ajuste significativo dos coeficientes. Os meses de março, abril, junho e o período anual apresentaram apenas um coeficiente de ajuste significativo, a 5 ou a $1 \%$ de probabilidade, enquanto os coeficientes restantes foram não significativos. Porém, tanto para estes como para os demais meses, todos os coeficientes $\beta$ foram mantidos no modelo, por colaborarem com a qualidade final do mesmo, visto que a retirada dos não-significativos ocasionou redução do coeficiente de determinação e do RMS. Segundo Ferreira (2005), este procedimento pode ser empregado sem prejuízo significativo para a qualidade final dos modelos, visto que os graus de liberdade do resíduo são consideravelmente altos.

Em trabalho semelhante, Mello; Silva (2009), ao modelarem a precipitação em função das coordenadas geográficas e altitude para o estado de Minas Gerais, também obtiveram modelos com características estatísticas de precisão semelhantes, com $\mathrm{R}^{2}$ máximo de 0,802 . Os autores concluíram que os modelos estatísticos podem ser aplicados e apresentam um erro relativamente baixo, em se tratando da modelagem de variável física ambiental, a qual apresenta variabilidade naturalmente alta em virtude do grande número de atributos envolvidos na sua dinâmica, cuja inclusão destes em um modelo estatístico tornaria sua aplicação prática bastante complexa. Tal conclusão também pode ser aplicada aos resultados advindos do presente trabalho.

Com relação às equações de $4^{\mathrm{a}}$ ordem estabelecidas (de melhor ajuste), geraram-se gráficos de comparação entre os valores observados e estimados e a correlação (r) entre eles (Figura 3). Em geral, a relação entre os valores observados e estimados pela regressão foram satisfatórios, sendo que os valores estimados se aproximaram significativamente aos dados observados. Percebe-se uma maior quantidade de pontos acima da linha 1:1, o que indica uma leve tendência de subestimativa dos modelos. As exceções são os meses de janeiro, fevereiro, junho e dezembro, que apresentaram pontos mais distribuídos ao redor da linha 1:1 (menor dispersão).

As dispersões residuais (diferença entre a precipitação observada e predita) representam o quanto a regressão polinomial de $4^{\mathrm{a}}$ ordem superestimou ou subestimou a precipitação. Na Figura 4 estão apresentados os gráficos dos meses que apresentaram a menor e maior dispersão dos resíduos.

O mês de junho apresentou os menores resíduos, ou seja, a regressão foi mais precisa neste mês, errando para mais ou para menos apenas $4,11 \mathrm{~mm}$ de precipitação. A maior superestimativa para este mês foi de $11,17 \mathrm{~mm}$, enquanto que a maior subestimativa foi de $-9,12 \mathrm{~mm}$. Os meses de novembro, dezembro e a precipitação anual apesentaram os maiores RMS na estimativa das precipitações. Nestes, a regressão polinomial de $4^{\mathrm{a}}$ ordem é menos precisa, podendo ter resíduos de até 16,91; 17,24 e 109,95 mm de precipitação, respectivamente. Para o mês de novembro (mês em que os modelos apresentaram pior desempenho) foi observado a maior superestimativa $(55,76 \mathrm{~mm})$, enquanto que as maiores subestimativas foram observadas em janeiro e novembro (38,81 e $37,65 \mathrm{~mm}$, respectivamente).

Silva et al. (2011) testaram métodos de interpolação (IDW e krigagem) para estimar as precipitações mensais no Espírito Santo. Dentre os índices estatísticos utilizados pelos autores para avaliar o melhor método de interpolação estavam o índice c e o EPAM. Os autores encontraram índices c bons e muito bons em sua maioria, mas também encontraram ajustes com desempenho sofrível e péssimo, de acordo com a classificação proposta por Camargo; Sentelhas (1997). Para o melhor método de interpolação (krigagem ordinária com modelo linear) o índice c variou entre 0,56 e 0,93. Já o modelo polinomial de $4^{\mathrm{a}}$ ordem apresentou índices c entre 0,71 e 0,92 e, ainda, demonstrou melhor desempenho em todos os meses, quando comparado à krigagem ordinária, com modelo linear.

Em relação ao EPAM, Silva et al. (2011) encontraram valores entre $7,9 \%$ e $14,6 \%$ para a krigagem ordinária com modelo linear. Já o modelo polinomial de $4^{\mathrm{a}}$ ordem apresentou erros entre $5,86 \%$ e $16,57 \%$. Somente nos meses de fevereiro, 
julho, agosto e setembro o desempenho do modelo polinomial de $4^{a}$ ordem foi inferior ao da krigagem ordinária com modelo linear. Percebe-se que a modelagem matemática dos totais mensais e anual precipitado por meio de regressões polinomiais múltiplas tem grande potencial como ferramenta preditora, pelo fato de gerarem estimativas com erros aceitáveis quando se considera uma variável meteorológica de grande variabilidade espacial como a precipitação.
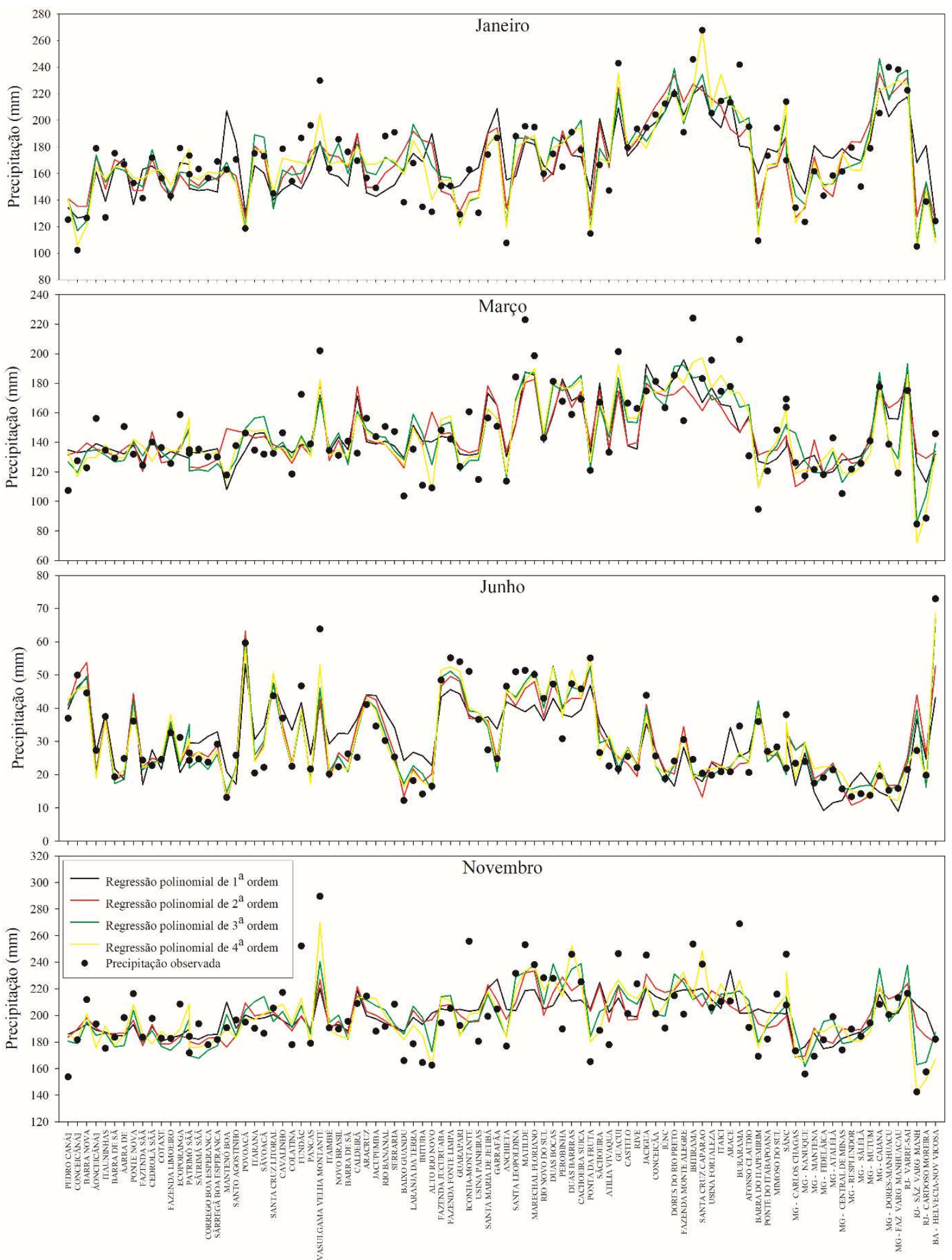

Postos pluviométricos

Figura 2. Modelos de regressão polinomial para os meses com melhor desempenho (junho), pior desempenho (novembro) e desempenhos intermediários (janeiro e março).

Figure 2. Polynomial regression models for the months with best performance (June), worst performance (November) and intermediate performances (January and March). 

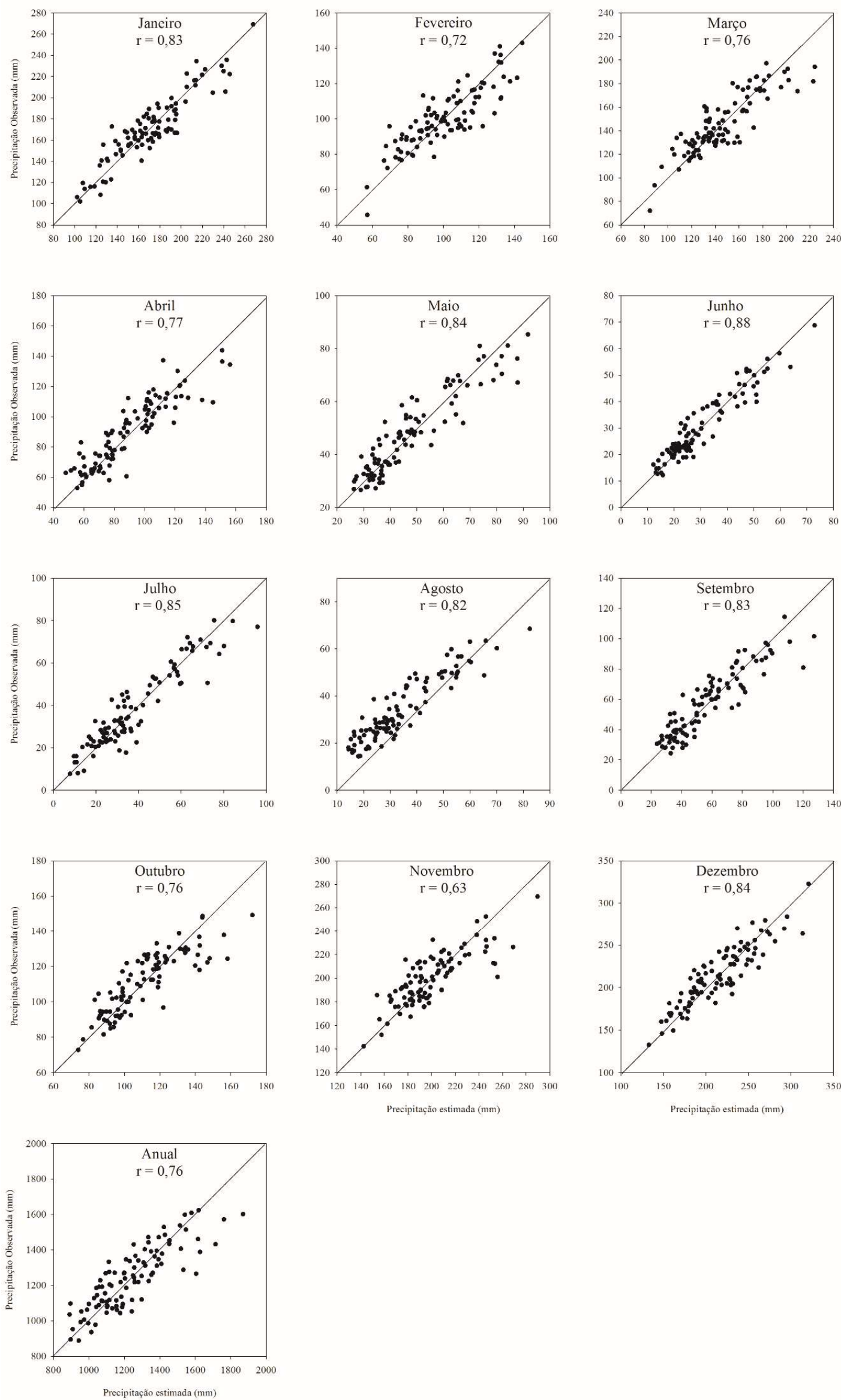

Figura 3. Correlação (r) entre os valores de precipitações mensais observados e estimados para o estado do Espírito Santo, pela regressão polinomial de $4^{\mathrm{a}}$ ordem. 
Figure 3. Correlation (r) between the values of monthly rainfall observed and estimated for the state of Espírito Santo, by the 4th-order polynomial regression.
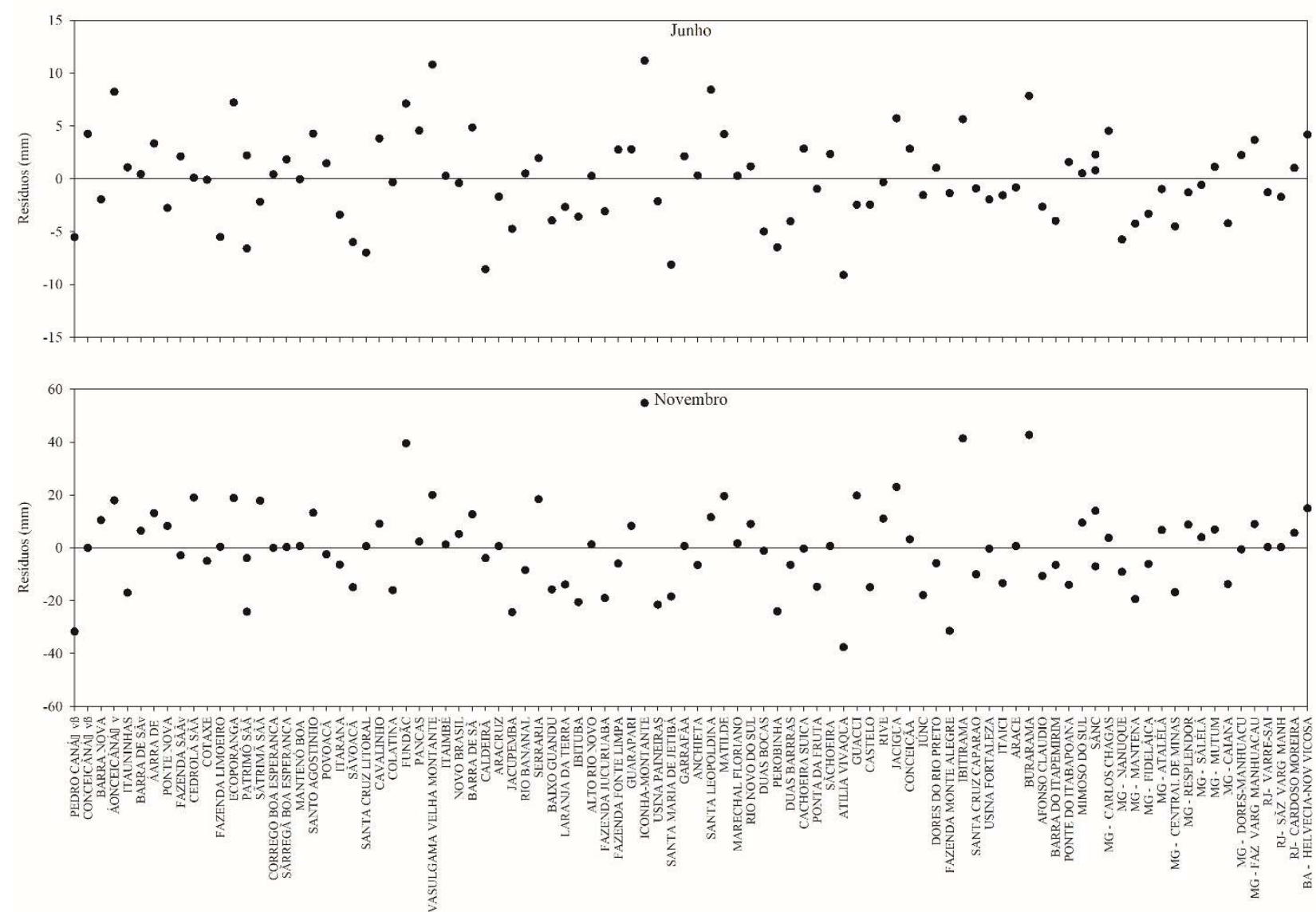

Figura 4. Dispersão dos resíduos gerados pela regressão polinomial de $4^{\mathrm{a}}$ ordem.

Figure 4. Scatter plot of the residuals generated by the 4 th order polynomial regression.

\section{CONCLUSÕES}

De acordo com a análise descritiva dos dados pluviométricos, o Espírito Santo apresenta grande variabilidade espacial da precipitação, sendo que as maiores variações se encontram nos meses de menor precipitação, entre junho e agosto.

A regressão polinomial que melhor se ajustou aos dados observados de precipitações médias mensais e precipitação média anual foi a de $4^{\mathrm{a}}$ ordem, apresentando os maiores coeficientes de determinação e menores dispersões residuais dos valores estimados.

A comparação entre os valores observados e estimados pela regressão polinomial de $4^{\mathrm{a}}$ ordem revelaram um ajuste eficiente dos dados pluviométricos na região estudada. Para os meses entre maio e agosto, a regressão apresentou as menores dispersões dos dados estimados e uma melhor precisão dos mesmos.

\section{AGRADECIMENTOS}

Os autores agradecem ao CNPq bela concessão de bolsas IC e PQ.

\section{REFERÊNCIAS}

ANA_AGÊNCIA NACIONAL DE ÁGUAS. Sistema de Informações Hidrológicas - HIDROWEB. 2018. Disponível em: <http://www.hidroweb.ana.gov.br/>. Acesso em: 03/01/2018.
ALVARES, C. A.; STAPE, J. L.; SENTELHAS, P. C.; GONÇALVES, J. L. DE M.; SPAROVEK, G. Köppen's climate classification map for Brazil. Meteorologische Zeitschrift, Berlin, v. 22, n. 6, p. 711-728, 2013. DOI: https://dx.doi.org/10.1127/0941-2948/2013/0507

ARAI, F. K.; GONÇALVES, G. G. G.; PEREIRA, S. B.; COMUNELlO, É.; VITORINO, A. C. T.; DANIEL, O. Espacialização da precipitação e erosividade na bacia hidrográfica do rio Dourados - MS. Engenharia Agrícola, Jaboticabal, v. 30, n. 5, p. 922-931, 2010. DOI: http://dx.doi.org/10.1590/S0100-69162010000500014

ASARIAN, J. E.; WALKER, J. D. Long-Term Trends in Streamflow and Precipitation in Northwest California and Southwest Oregon, 1953-2012. Journal of the American Water Resources Association, Herndon, v. 52, n. 1, p. 241-261, 2016. DOI: https://dx.doi.org/10.1111/17521688.12381

CASTRO, F. DA S.; PEZZOPANE, J. E. M.; CECÍLIO, R. A.; PEZZOPANE, J. R. M.; XAVIER, A. C. Avaliação do desempenho dos diferentes métodos de interpoladores para parâmetros do balanço hídrico climatológico. Revista Brasileira de Engenharia Agrícola e Ambiental, Campina Grande, v. 14, n. 8, p. 871-880, 2010. DOI: http://dx.doi.org/10.1590/S1415-43662010000800012

CAMARGO, A. P; SENTELHAS, P. C. Avaliação do desempenho de diferentes métodos de estimativas da evapotranspiração potencial no Estado de São Paulo, 
Brasil. Revista Brasileira de Agrometeorologia, Santa Maria, v. 5, n. 1, p. 89-97, 1997.

FERREIRA, D. F. Estatística básica. Lavras: UFLA, 2005. $760 p$.

GOMES, O. M.; SANTOS, C. A. C. DOS; SOUZA, F. de A. S. DE; PAIVA, W. DE; OLINDA, R. A. DE. Análise Comparativa da Precipitação no Estado da Paraíba Utilizando Modelos de Regressão Polinomial. Revista Brasileira de Meteorologia, São Paulo, v. 30, n. 1, p. 47 58, 2015. DOI: http://dx.doi.org/10.1590/0102778620120454

KELLER FILHO, T.; ASSAD, E. D.; LIMA, P. R. S. de R. Regiões pluviometricamente homogêneas no Brasil. Pesquisa Agropecuária Brasileira, Brasília, v. 40, p. 311-322, 2005

LI, Z.; NING, T.; LI, J.; YANG, D. Spatiotemporal variation in the attribution of streamflow changes in a catchment on China's Loess Plateau. CATENA, Amsterdam, v. 158, p. $1-8$, 2017.

DOI: https://dx.doi.org/10.1016/j.catena.2017.06.008

LOPES, I.; LEAL, B. G.; RAMOS, C. M. C.; MELO, J. M. M. DE. Espacialização da precipitação para a região do submédio São Francisco. Revista Brasileira de Agricultura Irrigada, Fortaleza, v. 10, n. 5, p. 893-903, 2016. DOI: http://dx.doi.org/10.7127/rbai.v10n500459

LYRA, G. B.; CORREIA, T. P.; OLIVEIRA-JÚNIOR, J. F. DE; ZERI, M. Evaluation of methods of spatial interpolation for monthly rainfall data over the state of Rio de Janeiro, Brazil. Theoretical and Applied Climatology, Wien, v. 134, n. 3-4, p. 955-965, 2017. DOI: https://dx.doi.org/10.1007/s00704-017-2322-3

MARQUÍNEZ, J.; LASTRA, J.; GARCÍA, P. Estimation models for precipitation in mountainous regions: the use of GIS and multivariate analysis. Journal of Hydrology, Amsterdam, v. 270, n. 1-2, p. 1-11, 2003. DOI: https://dx.doi.org/10.1016/S0022-1694(02)00110-5.

MELLO, C. R. DE; VIOLA, M. R.; CURI, N.; SILVA, A. M. DA. Distribuição espacial da precipitação e da erosividade da chuva mensal e anual no Estado do Espírito Santo. Revista Brasileira de Ciência do Solo, Viçosa, v. 36, n. $6, \quad$ p. $1878-1891, \quad 2012$. DOI: http://dx.doi.org/10.1590/S0100-06832012000600022

MELLO, C. R. DE; SILVA, A. M. DA. Modelagem estatística da precipitação mensal e anual e no período seco para o estado de Minas Gerais. Revista Brasileira de Engenharia Agrícola e Ambiental, Campina Grande, v. 13, n. 1, 2009. DOI: http://dx.doi.org/10.1590/S141543662009000100010

MELLO, C. R. DE; SÁ, M. A. C. DE; CURI, N.; MELLO, J. M. DE; VIOLA, M. R.; SILVA, A. M. DA. Erosividade mensal e anual da chuva no Estado de Minas Gerais. Pesquisa Agropecuária Brasileira, Brasília, v. 42, n. 4, p. 537-545, 2007. DOI: http://dx.doi.org/10.1590/S0100204X2007000400012
MELO JÚNIOR, J. C. F de; SEDIYAMA, G. C.; FERREIRA, P. A.; LEAL, B. G.; MINUSI, R. B. Distribuição espacial da freqüência de chuvas na região hidrográfica do Atlântico, Leste de Minas Gerais. Revista Brasileira de Engenharia Agrícola e Ambiental, Campina Grande, v. 10, n. 2, p. 417-425, 2006.

MINUZZI, R. B.; SEDIYAMA, G. C.; BARBOSA, E. M.; MELO JUNIOR, J. C. F. Climatologia do comportamento do período chuvoso da região sudeste do Brasil. Revista Brasileira de Meteorologia, São Paulo, v. 22, n. 3, p.338344, 2007. DOI: https://dx.doi.org/10.1590/S010277862007000300007

MINUZZI, R. B.; SEDIYAMA, G. C.; COSTA, J.M.N; VIANELLO, R.L.; Influência do fenômeno climático El Nino no período chuvoso da região sudeste do Brasil. Geografia, Londrina, v. 15, n. 2, jul./dez. 2006.

PIMENTEL-GOMES, F. Curso de Estatística Experimental. 12. ed. Piracicaba: Livraria Nobel, 1985. $467 \mathrm{p}$.

RANJITKAR, S.; SUJAKHU, N. M.; MERZ, J.; KINDT, R.; XU, J.; MATIN, M. A.; ALI, M.; ZOMER, R. J. Suitability Analysis and Projected Climate Change Impact on Banana and Coffee Production Zones in Nepal. PLOS ONE, SAn Francisco, v. 11, n. 9, p. e0163916, 2016. DOI: https://dx.doi.org/10.1371/journal.pone.0163916

SANT'ANNA NETO, J. L. Decálogo da climatologia do sudeste brasileiro. Revista Brasileira de Climatologia, Curitiba, v. 1, n. 1, p. 43-60, 2005.

SILVA, K. R. DA; CECÍlIO, R. A.; XAVIER, A. C.; PEZZOPANE, J. R. M.; GARCIA, G. DE O. Interpolação espacial da precipitação no Estado do Espírito Santo. Floresta e Ambiente, Seropédica, v. 18, n. 4, p. 417-427, 2011. DOI: http://dx.doi.org/10.4322/floram.2011.061

ULIANA, E. M.; REIS, E. F. DOS; SILVA, J. G. F. DA; XAVIER, A. C. Precipitação Mensal e Anual Provável para o Espírito Santo. Irriga, Botucatu, v. 18, n. 1, p. 139, 2013.

DOI: http://dx.doi.org/10.15809/irriga.2013v18n1p139

ZARO, G. C.; RICCE, W. DA S.; CARAMORI, P. H.; CARVALHO, S. L. C.; VICENTINI, M. E. Zoneamento agroclimático para a cultura do abacateiro no Estado do Paraná. Revista Brasileira de Fruticultura, Jaboticabal, v. 36 , n. 2, p. 363-372, 2014. DOI: http://dx.doi.org/10.1590/0100-2945-286/1 